\title{
Occlusal Plane Changes After Molar Distalization With a Pendulum Appliance in Growing Patients with Class II Malocclusion: A Retrospective Cephalometric Study
}

\author{
Marco Serafin' (D), Rosamaria Fastuca (D), Elisabetta Castellani ${ }^{3}$ (D), Alberto Caprioglio' (D)
}

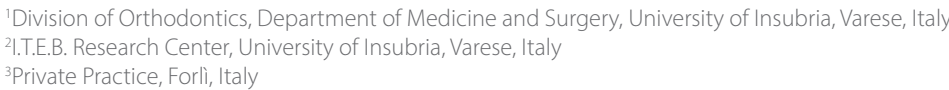

Main points:

- This study aimed to demonstrate the efficacy of pendulum appliance in distalizing the maxillary molars to correct a Class II malocclusion and provide a propaedeutic molar relationship before the final correction of the occlusion with fixed orthodontic treatment.

- A noteworthy result was the concordance of the data of this study with those of the previous ones; this emphasizes the environmental protocol and provides a further starting point for future investigations for the use of the pendulum appliance.

- Of particular interest was the maintenance of the occlusal plane inclination in the distalizing phase that could be achieved by the pendulum appliance compared with other distalizing techniques that do not allow a vertical control of the maxillary molars with consequent change in the occlusal plane and bite opening

- The absence of changes in the inclination of the occlusal plane seems to not interfere with the normal maxillary and mandibular growth, which could be a strategy to achieve an improved Class II correction.

\section{ABSTRACT}

Objective: This study aimed to evaluate the skeletal and dental changes after distalization with a pendulum appliance in growing patients with Class II malocclusion, focusing on the occlusal plane (OP).

Methods: The sample included 24 patients with Class II malocclusion (10 boys, 14 girls); their mean age was 12.1 years. All patients underwent molar distalization and had 2 serial cephalograms traced at baseline (T1) and after distalization (T2). Angular and linear dental changes were calculated by taking the sella-nasion (SN), palatal plane (PP), and pterygoid vertical as reference. OP inclination was compared with SN, $\mathrm{PP}$, and mandibular plane. The collected data were computed for all the tested variables, and one-way paired t-test was used to assess the significance of the differences between the time points. $\alpha$ was set at 0.05 . Multiple linear regressions were used to predict the OP changes.

Results: The mean total treatment time was $8 \pm 2$ months to obtain a super Class I molar relationship. In T1-T2 interval, statistically significant incisor buccal tipping of $5^{\circ} \pm 3.6^{\circ}(p<0.05)$, first molar distal tipping of $8.9^{\circ} \pm 8.3^{\circ}(p<0.001)$, and second molar tipping of $8.2^{\circ} \pm 8.1^{\circ}(p<0.001)$ were observed. The maxillary first and second molars moved significantly backward by $2.8 \pm 3.2 \mathrm{~mm}(p<0.05)$ and $3.7 \pm 2.7 \mathrm{~mm}(p<0.001)$, respectively. Only the premolars showed a statistically significant anchorage loss of $2.7 \pm 3.3 \mathrm{~mm}(p<0.05)$; overjet increased significantly at $1.3 \pm 1.2 \mathrm{~mm}(p<0.05)$. Regarding the $O P$, none of the tested variables showed any statistically significant changes between T1-T2.

Conclusion: The pendulum appliance showed efficacy in distalizing the maxillary first and second molars at the expense of anterior anchorage loss. The OP did not show statistically significant changes after molar distalization.

Keywords: Cephalometry, Class II Angle malocclusion, molar distalization, occlusal plane, pendulum appliance

\section{INTRODUCTION}

Class II malocclusion is one of the most frequent orthodontic problems, which dentists diagnose and treat, with a mean worldwide distribution of $19.56 \%$ in permanent dentition and $23.11 \%$ in mixed dentition (1). Depending on the diag-

Address for Correspondence: Marco Serafin, Division of Orthodontics, Department of Medicine and Surgery, University of Insubria, Varese, Italy E-mail: marco.serafin.1990@gmail.com

(C) Copyright 2020 by Turkish Orthodontic Society - Available online at turkjorthod.org
Received: April 28, 2020 Accepted: October 12, 2020 Available Online Date:

February 23, 2021 
nosis, Class II malocclusions can be treated by several methods, for example, functional orthopedic appliances, extraoral and intraoral devices for tooth distalization, camouflage treatment, or orthognathic surgery. If there are no significant skeletal discrepancies and tooth crowding, maxillary prognathism that involves Class II molar relationship can be completely corrected by maxillary molar distalization.

Molar distalization is a generally used non-extraction treatment to establish a Class I molar and canine relationship, especially using non-compliance devices (2). In fact, although the majority of intraoral and extraoral devices are effective for achieving molar distalization, they are often highly dependent on patient compliance. Among several intraoral distalizing devices, since its development in 1992, the pendulum appliance might be considered among one of the most investigated non-compliance and efficient tools in compensating a Class II molar relationship, especially those related with maxillary prognathism $(3,4)$.

The occlusal plane (OP) is a cephalometric line connecting the point bisecting the first molar cusp height and the point bisecting the overbite (5). Many factors change this plane inclination during craniofacial growth to achieve a harmonic function of the masticatory system. The OP reduces its inclination with respect to the maxilla and anterior cranial bases owing to an anterior rotation taking place during growth; this phenomenon is caused by a larger eruption of the upper molars than upper incisors (6). The cant of the OP is usually related to a malocclusion describing a vertical morphologic feature, which then affects the sagittal-mandibular position. Class III shows a flat OP, whereas Class II shows a steep one. Furthermore, orthodontic treatment changes the position and angulation of the involved teeth, and it is acknowledged that slight dental angular variations can result in significant alteration of the occlusion (7).

From a clinical perspective, treatment of the sagittal component of malocclusions, that is, distalizing techniques, could aim at changing the vertical position of the posterior teeth and subsequently the OP inclination and potentially advance the mandible toward a corrective position (8). OP is often strictly related to treatment, and a great part of the treatment effort is involved with its correction. A correct plane of occlusion allows protrusion without posterior interferences, enabling good function between the maxilla and mandible, whereas uncontrolled changes in the OP might lead to worsening of the malocclusion in some cases or difficulties in performing desired movements.

Several studies have evaluated the cephalometric changes after distalization with a pendulum from different perspectives (9). Nevertheless, the OP changes after distalization in Class II malocclusion are still not completely clarified, and this aspect is relevant. This study aimed to evaluate the skeletal and dental changes after upper molar distalization with a pendulum device in terms of potential changes in OP inclination in growing patients with a Class II malocclusion.

\section{METHODS}

\section{Sample Selection and Exclusion Criteria}

Ethical approval was obtained from the ethics committee of the University of Insubria (\#18/2014); the protocol followed adhered to the World Medical Association Declaration of Helsinki. Before starting treatment, an informed consent for releasing radiological diagnostic records for scientific research was obtained. Because the primary purpose of this study was to evaluate the effectiveness of molar distalization and its correlation with possible changes of the OP variables, the sample size was calculated on the cephalometric values of 5 patients, selecting the distance between the upper first molar centroid and the pterygoid vertical (PtV-Ctr1M) changes before and after distalization (mean=-2.7; standard deviation $[S D]=3.2$ ) as the main outcome. A sample size of at least 23 subjects was necessary to retrieve a power of 0.8 $(\beta=0.20)$ with a set at 0.05 . An initial sample of 30 patients was selected according to the following inclusion criteria:

1) Skeletal Class I or mild Class II malocclusion (ANB $\leq 6^{\circ}$ )

2) Bilateral Class II molar relationship

3) Sella-nasion (SN)-mandibular plane (MP) $<37^{\circ}$

4) Unerupted or incompletely erupted second maxillary molars

5) No previous orthodontic treatment and non-extraction treatment

6) Use of the pendulum appliance

7) High-quality radiographs with clear landmark visualization

Table 1. Sample selection and exclusion criteria

Initial sample

Primary exclusion criteria

1) Poor film quality

2) Incomplete records

Secondary exclusion criteria

1) Treatment time $>12$ months 3

Final sample 24

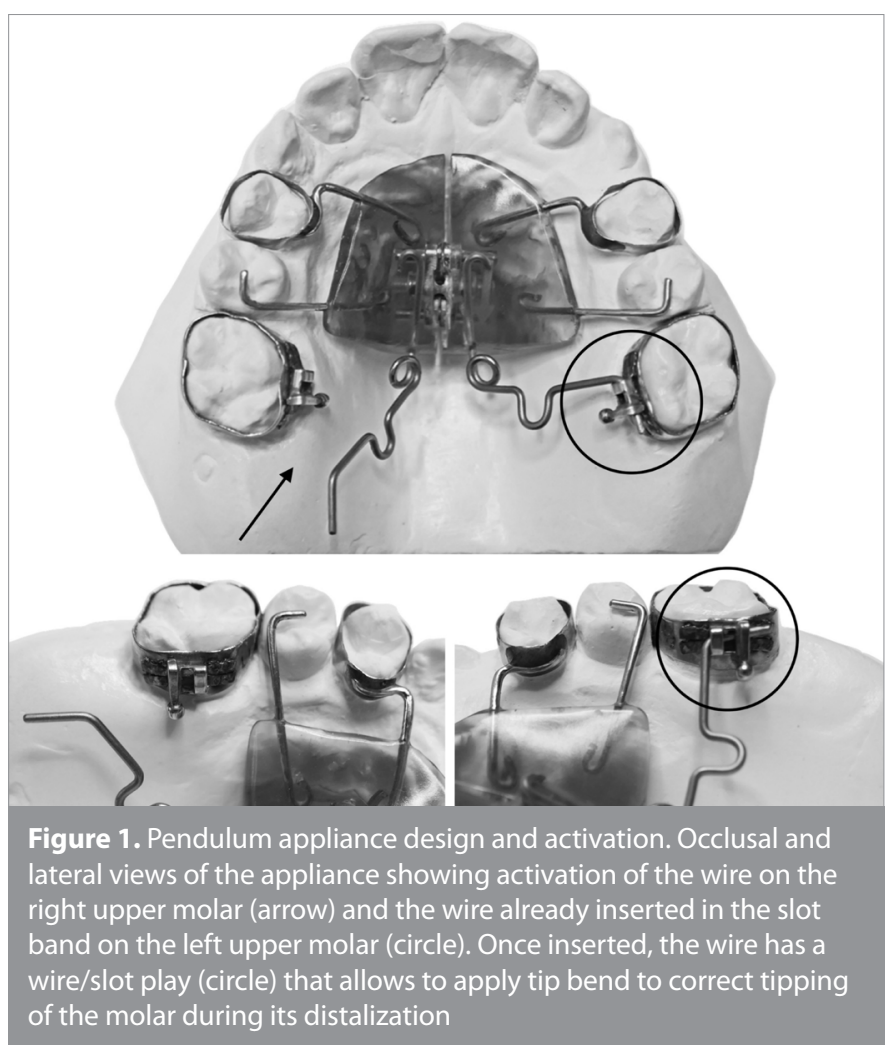


A total of 6 patients were excluded according to defined criteria as summarized in Table 1 . The final sample included 24 patients, 10 boys and 14 girls (mean age of 12.1 years; range, 10.5-14.2 years) with 2 serial cephalograms available at the observation times: baseline (T1) and after molar distalization (T2).

\section{Clinical Management}

In this study, a modified pendulum appliance was used with first molars bands, occlusal rests on premolars, and titanium-molybdenum alloy (TMA) springs (Figure 1 ); $45^{\circ}$ to $60^{\circ}$ activation angle of the TMA springs was customized on model casts to exert 200 to $250 \mathrm{~g}$. Owing to compensation of the palatal and coronal forces applied toward the center of resistance, uprighting bends $\left(25^{\circ}-30^{\circ}\right)$ were added to the end of the TMA wire to avoid excessive molar tipping; toe-in bends were also added (10-12). The pendulum was left in situ until a super Class I molar relationship was achieved.

\section{Cephalometric Analysis}

Lateral cephalograms were traced by a dedicated software (DeltaDent; Outside Format, Cremona, Italy) by the first operator (CE) with verification of anatomy and landmarks by the second one (SM). In case of overlapping structures (for example, gonial angle and teeth), a single averaged tracing was made. A conventional cephalometry, including skeletal and dental measurements, was used. Initial cephalometric measures at T1 are summarized in Table 2 .

The centroid point was obtained as the midpoint between the greatest mesial and distal convexity of the crowns of the maxillary first and second molars and the first premolars; the incisor tip was pointed at the incisal edge. The lines passing through the centroid and furca points and through the centroid/tip and apex were used as molar and premolar/incisor axes, respectively. SN (anterior cranial base plane), palatal plane (PP) (plane formed by the line connecting the anterior and posterior nasal spine), MP (plane formed by connecting gonion to gnathion points), and

Table 2. Mean and SD of cephalometric measurements at T1 and T2 and statistical comparison (paired t-test) with p values of T2-T1 period

\begin{tabular}{|c|c|c|c|c|c|c|c|}
\hline \multirow[b]{2}{*}{ Cephalometric measurement } & \multicolumn{2}{|c|}{ T1 } & \multicolumn{2}{|c|}{ T2 } & \multicolumn{2}{|c|}{$\Delta \mathrm{T} 2-\mathrm{T} 1$} & \multirow[b]{2}{*}{$\mathbf{p}$} \\
\hline & Mean & SD & Mean & SD & Mean & SD & \\
\hline \multicolumn{8}{|l|}{ Skeletal } \\
\hline SNA $\left({ }^{\circ}\right)$ & 81.2 & 2.5 & 80.8 & 3.3 & -0.4 & 2.1 & NS \\
\hline SNB $\left(^{\circ}\right)$ & 77.4 & 2.9 & 77.8 & 3.1 & 0.4 & 2.1 & NS \\
\hline ANB $\left(^{\circ}\right)$ & 3.6 & 1.8 & 3.1 & 1.7 & -0.5 & 1.3 & NS \\
\hline SN-PP $\left(^{\circ}\right)$ & 7.7 & 3.0 & 7.8 & 3.6 & 0.1 & 2.3 & NS \\
\hline $\operatorname{SN}-\mathrm{MP}\left({ }^{\circ}\right)$ & 30.6 & 3.8 & 31.4 & 4.6 & 0.8 & 3.0 & NS \\
\hline PtV-A (mm) & 55.5 & 2.8 & 55.6 & 3.1 & 0.1 & 2.1 & NS \\
\hline PtV-B (mm) & 55.3 & 4.4 & 56.1 & 4.7 & 0.7 & 2.8 & NS \\
\hline ANS-Me (mm) & 65.3 & 5.4 & 67.1 & 6.0 & 1.8 & 1.8 & NS \\
\hline \multicolumn{8}{|l|}{ Dental } \\
\hline SN-AxU1 ( $\left.{ }^{\circ}\right)$ & 103.2 & 8.3 & 108.2 & 8.1 & 5.0 & 3.6 & $<0.05$ \\
\hline SN-Ax1Pm ( $\left.{ }^{\circ}\right)$ & 84.0 & 4.5 & 86.5 & 6.3 & 2.5 & 5.1 & NS \\
\hline SN-Ax1M ( $\left(^{\circ}\right)$ & 67.9 & 5.1 & 59.0 & 9.6 & -8.9 & 8.3 & $<0.001$ \\
\hline $\mathrm{SN}-\mathrm{A} \times 2 \mathrm{M}\left({ }^{\circ}\right)$ & 60.9 & 3.2 & 52.7 & 4.8 & -8.2 & 8.1 & $<0.001$ \\
\hline PtV-TipU1 (mm) & 56.2 & 3.7 & 57.7 & 3.9 & 1.5 & 2.8 & NS \\
\hline PtV-Ctr1Pm (mm) & 40.2 & 3.3 & 42.8 & 4.3 & 2.7 & 3.3 & $<0.05$ \\
\hline PtV-Ctr1M (mm) & 22.3 & 3.5 & 19.5 & 3.6 & -2.8 & 3.2 & $<0.05$ \\
\hline PtV-Ctr2M (mm) & 13.1 & 3.1 & 9.4 & 2.7 & -3.7 & 2.7 & $<0.001$ \\
\hline PP-TipU1 (mm) & 29.0 & 3.0 & 29.5 & 3.5 & 0.5 & 1.4 & NS \\
\hline PP-Ctr1Pm (mm) & 21.0 & 2.8 & 22.3 & 3.2 & 1.4 & 1.8 & NS \\
\hline PP-Ctr1M (mm) & 17.5 & 2.8 & 17.4 & 2.7 & -0.1 & 1.6 & NS \\
\hline PP-Ctr2M (mm) & 12.1 & 4.6 & 12.2 & 4.0 & 0.1 & 2.4 & NS \\
\hline OVJ (mm) & 3.9 & 1.6 & 5.2 & 2.1 & 1.3 & 1.2 & $<0.05$ \\
\hline OVB (mm) & 3.2 & 1.9 & 2.8 & 2.1 & -0.4 & 1.9 & NS \\
\hline \multicolumn{8}{|l|}{$\mathrm{OP}$} \\
\hline SN-OP $\left({ }^{\circ}\right)$ & 19.8 & 4.3 & 19.3 & 3.9 & -0.5 & 2.3 & NS \\
\hline PP-OP $\left({ }^{\circ}\right)$ & 11.7 & 4.1 & 11.2 & 4.1 & -0.5 & 0.1 & NS \\
\hline OP-MP $\left(^{\circ}\right)$ & 15.8 & 3.1 & 16.8 & 4.3 & 1.0 & 3.4 & NS \\
\hline
\end{tabular}

NS: Not significant ( $p>0.05)$; SD: Standard deviation; Ax1M: Maxillary first molar axis; Ax2M: Maxillary second molar axis; OP: Occlusal plane; PP: Palatal plane; MP: Mandibular plane; SN: Sella-nasion plane; TipU1: Upper central incisor tip; Ctr1Pm: Maxillary first premolar centroid; Ctr1 M: Maxillary first molar centroid; Ctr2M: Maxillary second molar centroid; PP: Palatal plane; PtV: Pterygoid vertical; OVJ: Overjet; ANS: Anterior nasal spine; OVB: Overbite 
PtV (line perpendicular to the Frankfort plane at the posterior margin of the pterygomaxillary fissure) were used as horizontal and vertical reference planes for angular and linear parameters (Figures 2 and 3). OP was evaluated using the angular measurements of SN-OP, PP-OP, and OP-MP as shown in Figure 2.

\section{Statistical Analysis}

Descriptive statistics was calculated for cephalometric measurements at T1 and T2 for all the subjects. Mean differences and SDs

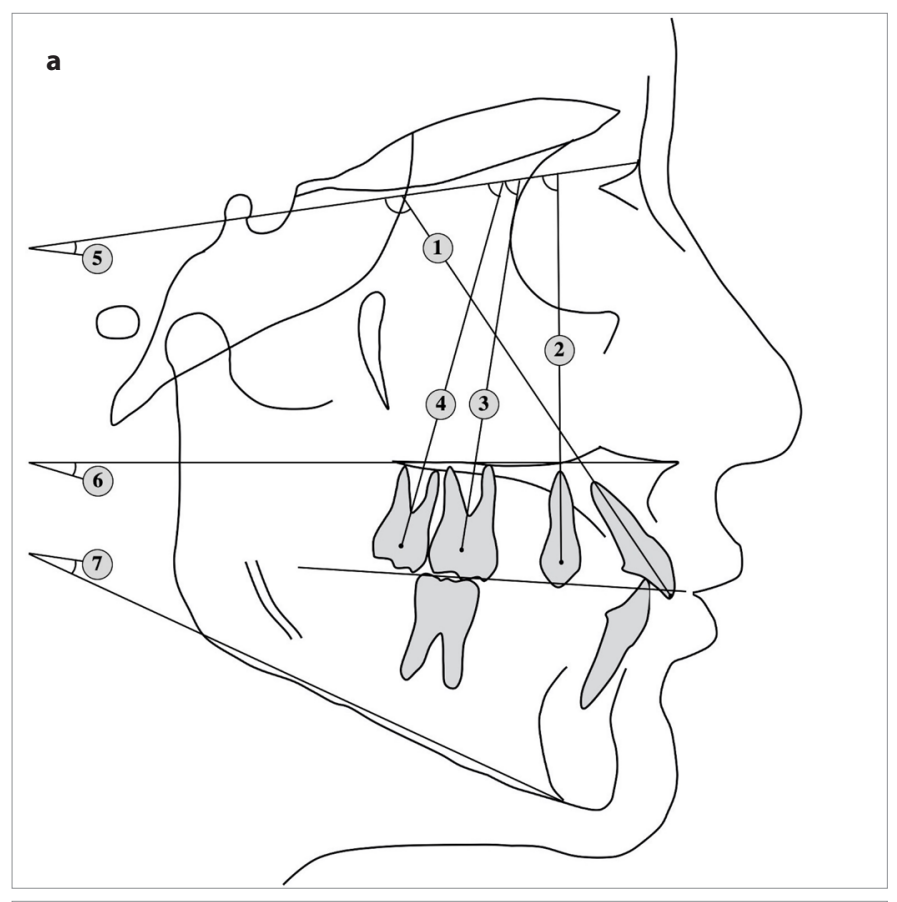

b

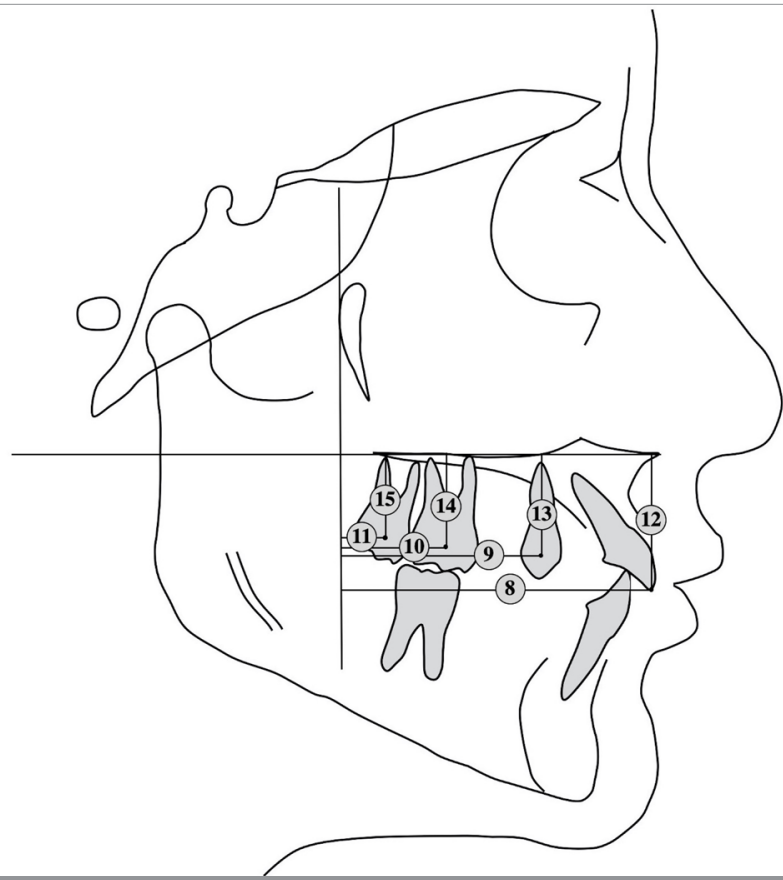

Figure 2. a, b. (a) Cephalometric analysis for evaluation of maxillary tooth and occlusal plan changes. 1) SN-AxU1; 2) SN-Ax1Pm; 3) SNAx1M; 4) SN-Ax2M; 5) SN-OP; 6) PP-OP; 7) MP-OP (b) Cephalometric analysis of the evaluation of maxillary tooth and occlusal plane changes. 8) PtV-TipU1; 9) PtV-Ctr1Pm; 10) PtV-Ctr1M; 11) PtV-Ctr2M; 12) PP-TipU1; 13) PP-Ctr1Pm; 14) PP-Ctr1M; 15) PP-Ctr2M between T1 and T2 were also calculated. The Statistical Package for Social Sciences version 22.0 software (IBM Corp.; Armonk, NY, USA) was used for statistical analysis. The Shapiro-Wilk test confirmed the normal distribution of the data for the tested sample, and parametric tests were used to compare the mean differences among different timepoints. Data were computed for all the tested variables, and the paired t-test was used to assess significance of the differences among the timepoints. Significance level (p) was set at 0.05 .

The Pearson correlation coefficient ( $r$ ) was employed to evaluate the strength of the relationship between several tested parameter (SN-MP; SN-Ax1M; SN-Ax2M; PtV-Ctr1M; PtV-Ctr2M; PP-Ctr1M; and PP-Ctr2M) changes after distalization and the changes in SN-OP. Finally, the multiple backward linear regressions were used to estimate the association of each tested parameter with the SN-OP changes.

\section{Method Error}

The method error was quantified with the method of moments variance estimator. A total of 10 lateral cephalograms were randomly selected and retraced by a third orthodontist (CA). A paired t-test showed no significant differences between the 2 series of cephalometry. The mean error and $95 \%$ confidence interval among the repeated data were $0.7(0.5-0.8) \mathrm{mm}$ for linear measurements and $0.8^{\circ}\left(0.6^{\circ}-0.9^{\circ}\right)$ for angular measurements; reliability coefficient $(r)$ for linear and angular measurement ranged from $94 \%$ to $98 \%$ and from $92 \%$ to $97 \%$, respectively.

\section{RESULTS}

The mean treatment time from the maxillary molar distalization to achieving a super Class I was $8 \pm 2$ months. Mean and SD and paired t-test results of the skeletal, dental, and occlusal changes are reported in Table 2 . The most significant changes are reported below.

\section{Skeletal Changes}

Changes in skeletal measurements were observed. SNA (Sella-Nasion-Point A) decreased $0.4^{\circ} \pm 2.1^{\circ}$ and SNB (Sella-Nasion-Point B) increased $0.4^{\circ} \pm 2.1^{\circ}$; PtV-A (Pterygoid Vertical-Point A) and PtV-B (Pterygoid Vertical-Point B) increased $01 \pm 2.1 \mathrm{~mm}$ and $0.7 \pm 2.8 \mathrm{~mm}$, respectively. An increase in SN-MP (Sella-Nasion Plane-Mandibular Plane) of $0.8^{\circ} \pm 3^{\circ}$ accompanied by an increase in ANS-Me (Anterior Nasal Spine-Menton) of $1.8 \pm 1.8 \mathrm{~mm}$ was also observed. Inferential statistical analysis showed that none of these changes were significant ( $p>0.05)$.

\section{Dental Changes}

Statistically significant changes were observed in SN-Ax1M (Sella-Nasion plane-First maxillary molar axis), which decreased at $8.9^{\circ} \pm 8.3^{\circ}$ in the T1-T2 interval $(\mathrm{p}<0.001)$, whereas SN-Ax2M (Sella-Nasion plane-Second maxillary molar axis) insignificantly tipped distally by $8.2^{\circ} \pm 8.1^{\circ}$. SN-AxU1 (Sella-Nasion plane-Maxillary central incisor axis) also increased significantly at $5^{\circ} \pm 3.6^{\circ}$ after distalization $(p<0.05)$. Linear changes in teeth position in the case of PtV (Pterygoid Vertical) and PP (Palatal Plane) were observed. In the T1-T2 interval, the distances decreased by $2.8 \pm 3.2 \mathrm{~mm}$ for PtV- 
Ctr1M (Pterygoid Vertical-Maxillary first molar centroid) $(p<0.05)$ and $3.7 \pm 2.7 \mathrm{~mm}$ for PtV-Ctr2M (Pterygoid Vertical-Maxillary second molar centroid) $(p<0.001)$. Vertical extrusion was reported on all the analyzed teeth but without any statistically significant difference. OVJ (Overjet) increased significantly by $1.3 \pm 1.2 \mathrm{~mm}$ $(p<0.05)$. Both angular and linear changes were also observed on anchorage-loss teeth; the incisor and first premolars moved and tipped mesially, but only PtV-Ctr1 Pm (Pterygoid Vertical-Maxillary first premolar centroid) was statistically significant with a mesial movement of $2.7 \pm 3.3 \mathrm{~mm}(p<0.05)$. The main dental movements after molar distalization are resumed in Figure 4.

\section{Occlusal Plane Changes}

None of the tested variables related to the OP (Occlusal Plane) showed statistically significant differences between the 2 time-

Table 3. Results of the multiple backward linear regression to estimate association of the tested parameters with the SN-OP changes after distalization (T1-T2)

\begin{tabular}{|lccc} 
Explanatory variable & B & $\mathbf{t}$ & $\mathbf{P}$ \\
\hline SN-MP & 0.32 & 1.21 & 0.26 \\
SN-Ax1M & -0.21 & -0.65 & 0.53 \\
SN-Ax2M & -0.17 & -0.47 & 0.65 \\
PtV-Ctr1M & 0.02 & 0.06 & 0.95 \\
PtV-Ctr2M & 0.15 & 0.35 & 0.73 \\
PP-Ctr1M & -0.70 & -2.58 & 0.03 \\
PP-Ctr2M & -0.01 & -0.03 & 0.97
\end{tabular}

$\beta$ correlation coefficients; $R$ square of the model, 0.68 . Level of significance: $\mathrm{p}<0.05$.

MP: Mandibular plane; SN: Sella-nasion plane; Ax1M: Maxillary first molar axis; Ax2M: Maxillary second molar axis; Ctr1M: Maxillary first molar centroid; Ctr2M: Maxillary second molar centroid PP: Palatal plane; PtV: Pterygoid vertical

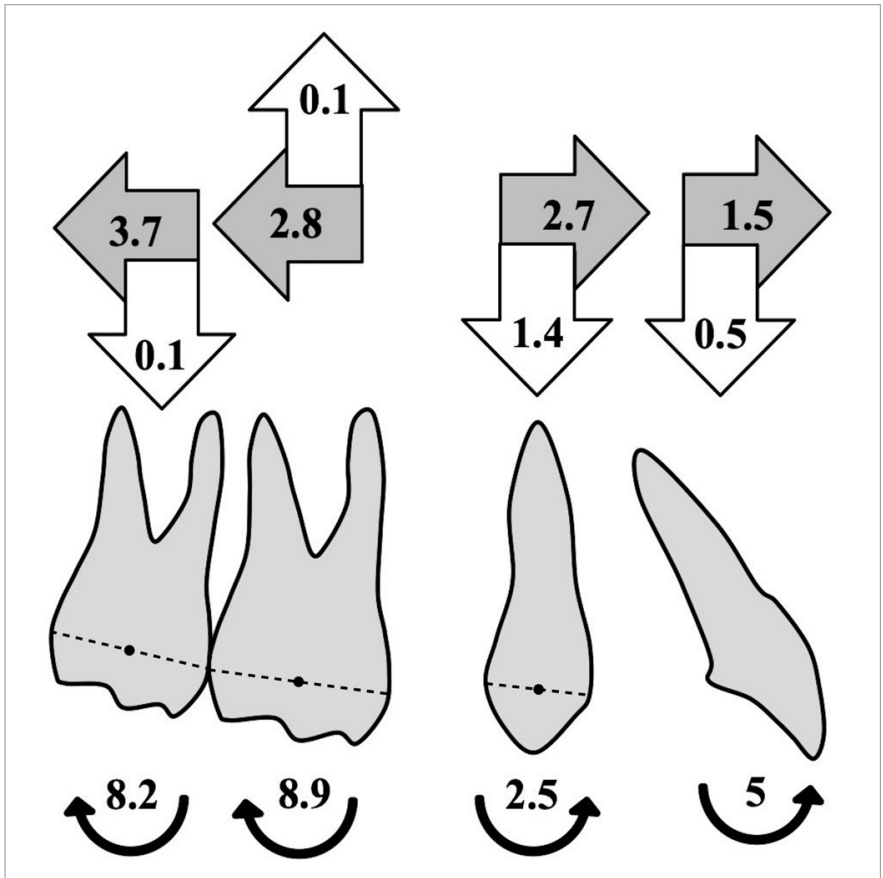

Figure 3. Main results after distalization of dentoalveolar measurements. Gray arrows, sagittal movements $(\mathrm{mm})$; white arrows, vertical movements $(\mathrm{mm})$; black arrows, mesiodistal (molars and premolar) and buccolingual (incisor) inclination movements $\left(^{\circ}\right)$ points; OP-MP (Occlusal Plane-Mandibular Plane) was increased by $1^{\circ} \pm 3.4^{\circ}$, whereas both SN-OP (Sella-Nasion plane-Occlusal Plane) and PP-OP (Palatal Plane-Occlusal Plane) showed a decrease of $0.5^{\circ} \pm 2.3^{\circ}$ and $0.5^{\circ} \pm 0.1^{\circ}$, respectively. In addition, results of multiple backward linear regressions (Table 3 ) showed that the tested parameters (SN-MP, SN-Ax1M, SN-Ax2M, PTV-Ctr1M, PTV-Ctr2M, PP-Ctr1M, and PP-Ctr2M) accounted for $68 \%$ of the changes in SN-OP after molar distalization. Moreover, none of the differences in the tested parameters were significantly associated with SN-OP changes in the multiple linear regressions.

\section{DISCUSSION}

This retrospective study described the result of 24 growing patients treated with a pendulum device. Pendulum is a largely studied non-compliance device, and it has been compared with similar tools that provide a combination of distal body movement and distal crown tipping (9). This phenomenon is accompanied by loss of anchorage and extrusion in the premolars and incisors. The mean treatment time for achieving a Class I molar relationship was about $8 \pm 2$ months, consistent with the treatment time of previous systematic reviews that report a range of 6 to 10 months, which probably depended on the full cusp or endto-end molar relationship of the sample before treatment (13). Several studies have also evaluated the influence of the pendulum in relation to the soft tissue and dentoskeletal improvement (13). The results of this study are assumed to be in accordance with the mean values of the previous studies on distalizing devices (14). However, till date, there are no studies regarding the use of the pendulum and its influence on the OP. Therefore, the purpose of this study was to evaluate the OP changes after maxillary molar distalization in growing patients.

\section{Skeletal Changes}

In terms of the skeletal changes, no statistical significance was found in the T1-T2 period. SNA decreased by $0.4^{\circ} \pm 2.1^{\circ}$, whereas SNB increased by $0.4^{\circ} \pm 2.1^{\circ}$ in T1-T2. PtV-A and PtV-B increased by $0.1 \pm 2.1 \mathrm{~mm}$ and $0.7 \pm 2.8 \mathrm{~mm}$, respectively. SNA reduction was like in other studies, corresponding more to a normal growth than any orthopedic influence of the appliance used (15). The pendulum causes primarily a dentoalveolar effect, but secondary mandibular growth can be essential for the correction of Class II malocclusion in growing patients (16). This provides a correction of the molar relationship because of molar distalization, whereas a favorable growth pattern drives the mandible forward as expected by the increase in SNB and PtV-B. Nevertheless, a period of approximately 8 months necessary for molar distalization seems to be too short to observe any remarkable and statistically significant cranial changes compared with the use of functional orthopedic appliances (17). Therefore, increase in the mandibular length was similar to the measurements reported in untreated patients and is variable according to mean treatment time (18).

The increase in SN-MP is often evaluated because of the risk of producing an anterior open bite (19). Class II correction by conventional molar distalization is not always suggested. It can be stated that mandibular rotation is related to changes in the dis- 
talization, corrective orthodontic mechanics, and craniofacial growth and development (20). In fact, in open bite patients, extrusion and distalization could assess a backward mandibular rotation, increasing the anterior lower facial height and resulting in a worse molar relationship. In this study, SN-MP was increased only by $0.8^{\circ} \pm 3^{\circ}$, and lower anterior face height was increased by $1.8 \pm 1.8 \mathrm{~mm}$, although without any statistical significance. The vertical increase in facial height seems to represent only a temporary effect, which could be completely compensated by a favorable counterclockwise mandibular growth (2). The variability of results in the literature may be related to differences in sample size, mean age, vertical facial pattern, and criteria used to classify the patients (21). Because of these considerations and to prevent bite opening, it has been speculated that distalization techniques are more suitable in patients with normal or reduced divergence, as was the case in the sample collected for our study.

\section{Dental Changes}

In this study, the mean distal shift of the first and second maxillary molars was $2.8 \pm 3.2 \mathrm{~mm}$ and $3.7 \pm 2.7 \mathrm{~mm}$, respectively. Previous studies have reported a mean molar distal movement ranging between 2 and $6.4 \mathrm{~mm}$, depending on the presence or absence of the upper second molar. Our results are also in accordance with the mean of $3.1 \mathrm{~mm}$ for palatal distalizing appliances described in previous studies $(9,14)$. The pendulum appliance can achieve a large amount of molar distalization, but it depends on many factors, the first being the eruption stage of second maxillary molars (22). The authors agreed with the idea that the presence of completely erupted second molars decreased the efficiency of distalization, increased the treatment time, required a higher force, and produced a larger anchorage loss but with less tipping than bodily movement of the first molar. The best time to start the pendulum treatment is when the second molars are unerupted, although more crown tipping can be expected (23). Owing to the mean age, the sample of this study had incompletely erupted second molars; therefore, distal tipping of the first maxillary molars seemed to be greater than bodily movement, probably because of the unerupted second molars, which acted as a distal crown fulcrum instead of the center of rotation.

It is important to define in detail what distal movement is in comparison with that in other studies on the basis of the reference point, for example, centroid of the radiological crown as used in this study or the tip of a cusp and the distance between it and the center of rotation changes. The closer the reference point is to the center of rotation, the smaller the amount of distal movement that will be obtained (24). Therefore, data will have to be carefully compared not only in terms of the reference point but also the reference plane.

The pendulum should ideally provide a bodily distal displacement, but distal tipping is often produced by distalizing appliances. Significant changes were observed in the first and second molar tipping that increased distally by $8.9^{\circ} \pm 8.3$ and $8.2 \pm 8.1^{\circ}$, respectively. A previous systematic review has reported a molar distal tipping from $8.4^{\circ}$ to $14.5^{\circ}(9)$. This shows that the pendulum is not effective in pure distalization. As mentioned earlier, the eruption stage of the second molars can be a discriminating factor in the tipping amount. Furthermore, the ratio between the reference point and the center of rotation must be considered. The uprighting bends on the TMA loop also can be a reason for a decrease in the final molar tipping. Therefore, comparison with other studies is not always possible without a strict similarity of mechanical and environmental conditions.

Upper molars, first premolars, and upper incisors have vertically changed insignificantly. The pendulum is used to prevent molar extrusion by rigid bonding and intrusive forces exerted by the tongue. Maxillary molar eruption at the mean age of 12 years during the 8 months of distalizing treatment did not show statistically significant changes. The first molars intruded $0.1 \pm 1.6 \mathrm{~mm}$ and second molars extruded $0.1 \pm 2.4 \mathrm{~mm}$ using PP as a reference line, and these values seem to be insignificant, especially in the bite opening (SN-MP), which was $0.8^{\circ} \pm 3^{\circ}$. The mean values reported in the literature show an intrusion variable from 0.1 to $1.7 \mathrm{~mm}$; it has been demonstrated that the pendulum does not create critical molar extrusion $(9,25)$. The design and activation of the modified pendulum provide an explanation for the result obtained because of the trajectory of the TMA loop, which contrasts with the normal eruption of the maxillary molar eruption during growth. The first premolars extruded $1.4 \pm 1.9 \mathrm{~mm}$ and incisors extruded $0.5 \pm 1.4 \mathrm{~mm}$; these results are comparable with the incisor extrusion caused by the patient's growth and are in accordance with the previous studies that report extrusion on the premolar and incisor region (14). An explanatory statement is that the appliance itself rotates the maxilla around its center of rotation between the molars and premolars but without changing the opening of the skeletal bite. This rotation helps to prevent an increase in the vertical dimension despite a modified pendulum promoting bodily distal movement and smaller distal tipping.

The major disadvantage of distalizing techniques, common to all intraoral tooth-bonded distalization devices, is the forward drift of the anchoring units (21). The resulting loss of anchorage is stated by an uncontrolled mesial movement of the medial and anterior segments. The first premolars anchorage loss found was $2.7 \pm 3.3 \mathrm{~mm}$ mesial movement and $2.5^{\circ} \pm 5.1^{\circ}$ mesial tipping. The mean distance obtained by molar distalization and anchorage loss was $5.5 \mathrm{~mm}$, and the premolar anchorage loss represents $49 \%$ of the space opened. The systematic revision used as reference indicates a range between $24 \%$ and $46 \%$ of anchorage loss (9); a greater anchorage loss is probably because of uprighting bends that had been added to the TMA springs. Another effect of this device is incisor anchorage loss; the forces transmitted to anterior teeth and the movements exercised by the activated springs cause a movement of the anchored incisors (2). The amount of anchorage loss of the incisors in this study was $1.5 \pm 2.8$ $\mathrm{mm}$ and $5^{\circ} \pm 3.6^{\circ}$. Throughout distalization, the upper incisors are proclined as a result of reaction forces that act first on the bonded premolars before being conducted to the incisor segment. Therefore, the pendulum could be more appropriate in Class II patients with a reduced incisor buccal inclination. Furthermore, the variability of results may be influenced by the early use of uprighting bends that provide a greater bodily movement, and the position of second molars could be an obstacle to first mo- 
lar distalization at the expense of anchorage loss and time (23). Skeletal anchorage could be a strategy to prevent anchorage loss with the use of an anchored pendulum.

\section{OP Changes}

Numerous authors have attempted to explain the factors responsible for a successful Class II treatment, which could be the changes in the level of occlusal line and growth. The cant of the OP also describes a vertical trait, which may affect the mandibular position and solve a skeletal discrepancy. In fact, changes in the inclination of the OP can, in part, compensate for unfavorable skeletal factors outside the bite to create a normal occlusion (26). To date, no study has analyzed the relationship between the use of pendulum and OP changes, although some data are available. There are only a few studies available to compare the OP changes $(3,19,20,27-29)$. There is a wide agreement between the longitudinal data reported in the literature and data of this study, showing progressive horizontalization of the occlusal plan followed by simultaneous reduction in the mandibular plane angle as the mandible adapts.

The line that forms the OP is influenced by molar and incisal vertical position. Changes in the vertical position of both maxillary and mandibular teeth result in changes in the OP; as a result, the definition of OP can be the first discriminating factor, for example, bisected or functional OP (30). In addition, changes in the OP could depend on the changes in the reference plane: SN, PP, and MP.

A comparison of changes in the OP inclination between treated and untreated patients should be made, comparing the normal changes observed in growing patients by age. It is reported that between the age of 12 and 13 years, and SN-OP, PP-OP, and OPMP have values of $19.4^{\circ}, 10^{\circ}$, and $15^{\circ}$, respectively (6). Our study reported corresponding values of $19.8^{\circ}, 11.7^{\circ}$, and $15.8^{\circ}$, respectively, at $\mathrm{T} 1$ and $19.3^{\circ}, 11.2^{\circ}$, and $16.8^{\circ}$, respectively, at $\mathrm{T} 2$, after a mean time of 8 months of distalization. It is interesting to observe the trend of changes of these values before and after the pubertal growth spurt; as reported, SN-OP and PP-OP decrease, whereas OP-MP increases constantly. On an average, a counterclockwise rotation of the mandible and OP is expected with age, implying a correlation between OP inclination and mandibular repositioning during growth and skeletal development (31).

These changes in the orientation of OP play an important role in the anterior rotation that takes place during growth. It has been also reported that the OP rotates upward and forward an average of $6.1^{\circ}$ between the ages of 6 and 16 years, which allows to manage and to possibly solve spontaneously a third of Class II discrepancies (7). As mentioned earlier, the pendulum itself rotates the maxilla around its center of rotation, whereas the maxillary teeth change their vertical position, and the combination of both events, concurrently normal growth of the dentofacial complex, does not seem to influence the natural tendency of the $\mathrm{OP}$ to rotate forward. The use of a distalizing appliance that does not influence the cant of the OP could be a strategy to not influence normal growth and favor not only dental but also skeletal correction of the Class II malocclusion.
PP-OP decreased $0.5^{\circ} \pm 0.1^{\circ}$ after molar distalization. A reduction in this angle was also observed in growing patients with Class II; nevertheless, these values seem to be less important in the craniofacial complex development (31). OP-MP could be more meaningful, more expressive of type, and more expressive on the vertical positions of the teeth within the dentofacial complex; this angle is a measure of relative posterior alveolar height and also correlates with SN-MP (32). In this study, OP-MP increased insignificantly by $1^{\circ} \pm 3.4^{\circ}$ after molar distalization, starting with a value of $15.8^{\circ} \pm 3.1^{\circ}$. The position of the occlusal line is determined principally by the vertical growth of both upper and lower teeth, and its inclination is defined mostly by the development of the dentoalveolar bone (31). It has been speculated that a control of extrusion in the mandibular arch prevents clockwise rotation of the mandible, which can lead to a worse vertical dimension and aggravate both skeletal and dental Class II discrepancies.

The cant of the OP is difficult to correlate with the changes produced by orthodontic treatment or normal growth, because it is necessary to consider many dental and skeletal values, including mandibular ones, and the function must also be considered. For clinicians, orthodontic treatment of the anteroposterior components of malocclusion could aim at changing the OP inclination, reducing it, possibly facilitating the adaptation of the mandible for an improved skeletal relationship with the maxilla (8). It is reasonable to assume that any variation in the occlusion would alter the jaw position in relation to the maxillary occlusal surfaces (31). Therefore, the main clinical implication could be to not tip the $\mathrm{OP}$ by raising the posterior end when a patient starts with high $\mathrm{SN}-\mathrm{MP}$ values to prevent bite opening.

The main limitation of this study was the absence of a control group to compare the OP changes; unfortunately, there were no previous studies that evaluated the correlation between Class II malocclusion and its potential correction related to OP changes. As a preliminary study, the authors did not include a control group. Further studies are needed with a control group for comparison. Another limitation of this study was the observation time of the sample; all the patients were treated with a fixed orthodontic appliance after a period of approximately 4 months after molar distalization and Nance button retention. It may be interesting to evaluate the changes in the OP in patients treated only with distalization and following the craniofacial growth. Limitations of this study also include the evaluation of 3D landmarks on bidimensional cephalograms and potential bias from converting bilateral structures to single outlines; hence, 3D analysis should be addressed in further studies.

\section{CONCLUSION}

The pendulum appliance was effective in distalizing the first and second maxillary molars with negligible extrusion, moderate distal tipping, and moderate anterior anchorage loss. The pendulum appliance seems to take advantage of its biomechanics in not producing significant changes in OP inclination. Further long-term and controlled studies are needed to compare the present data. 
Ethics Committee Approval: This study was approved by Ethics committee of University of Insubria of Varese (Italy), (Approval No: 18/2014).

Informed Consent: Written informed consent was obtained from the patients who agreed to take part in the study.

Peer-review: Externally peer-reviewed.

Author Contributions: Supervision - A.C.; Design - M.S., E.C.; Supervision - R.F., A.C.; Materials - E.C., A.C.; Data Collection and/or Processing - E.C., R.F.; Analysis and/or Interpretation - M.S., R.F.; Literature Search M.S., E.C.; Writing Manuscript - M.S.; Critical Review - R.F., A.C.

Conflict of Interest: The authors have no conflict of interest to declare.

Financial Disclosure: The authors declared that this study has received no financial support.

\section{REFERENCES}

1. Alhammadi MS, Halboub E, Fayed MS, Labib A, El-Saaidi C. Global distribution of malocclusion traits: A systematic review. Dental Press J Orthod 2018; 23: 40.e1-40.e10. [Crossref]

2. Fontana M, Cozzani M, Caprioglio A. Non-compliance maxillary molar distalizing appliances: an overview of the last decade. Prog Orthod 2012; 13: 173-84. [Crossref]

3. Ghosh J, Nanda RS. Evaluation of an intraoral maxillary molar distalization technique. Am J Orthod Dentofacial Orthop 1996; 110: 63946. [Crossref]

4. Hilgers JJ. The pendulum appliance for Class II non-compliance therapy. J Clin Orthod 1992; 26: 706-14.

5. Downs WB. Variations in facial relationships; their significance in treatment and prognosis. Am J Orthod 1948; 34: 812-40. [Crossref]

6. Vukusić $N$, Lapter M, Muretić Z. Change in the inclination of the occlusal plane during craniofacial growth and development. Coll Antropol 2000; 24: 145-50.

7. Braun S, Legan HL. Braun S, Legan HL. Changes in occlusion related to the cant of the occlusal plane. Am J Orthod Dentofacial Orthop 1997; 111: 184-8. [Crossref]

8. Čelar A, Tafaj E, Graf A, Lattner S. Association of anterior and posterior occlusal planes with different Angle and skeletal classes in permanent dentitions: A lateral cephalometric radiograph study. J Orofac Orthop 2018; 79: 267-76. [Crossref]

9. Antonarakis GS, Kiliaridis S. Maxillary molar distalization with noncompliance intramaxillary appliances in Class II malocclusion. A systematic review. Angle Orthod 2008; 78: 1133-40. [Crossref]

10. Kinzinger G, Syree C, Fritz U, Diedrich P. Molar distalization with different pendulum appliances: In vitro registration of orthodontic forces and moments in the initial phase. J Orofac Orthop 2004; 65: 389-409. [Crossref]

11. Kinzinger GSM, Wehrbein H, Diedrich PR. Molar distalization with a modified pendulum appliance - in vitro analysis of the force systems and in vivo study in children and adolescents. Angle Orthod 2005; 75: 484-93.

12. Kinzinger GSM, Diedrich PR. Biomechanics of a modified pendulum appliance - theoretical considerations and in vitro analysis of the force systems. Eur J Orthod 2007; 29: 1-7. [Crossref]

13. Al-Thomali Y, Basha S, Mohamed RN. Pendulum and modified pendulum appliances for maxillary molar distalization in Class II malocclusion - a systematic review. Acta Odontol Scand 2017; 75: 394401. [Crossref]

14. Kinzinger GS, Eren M, Diedrich PR. Treatment effects of intraoral appliances with conventional anchorage designs for non-compliance maxillary molar distalization: A literature review. Eur J Orthod 2008; 30: 558-71. [Crossref]

15. Mossaz CF, Byloff FK, Kiliaridis S. Cervical headgear vs pendulum appliance for the treatment of moderate skeletal Class II malocclusion. Am J Orthod Dentofacial Orthop 2007; 132: 616-23. [Crossref]

16. Burkhardt DR, McNamara JA Jr, Baccetti T. Maxillary molar distalization or mandibular enhancement: a cephalometric comparison of comprehensive orthodontic treatment including the pendulum and the Herbst appliances. Am J Orthod Dentofacial Orthop 2003; 123: 108-16. [Crossref]

17. Taylor KL, Evangelista K, Muniz L, Ruellas ACO, Vallarades-Neto J, McNamara J Jr, et al. Three-dimensional comparison of the skeletal and dentoalveolar effects of the Herbst and Pendulum appliances followed by fixed appliances: A CBCT study. Orthod Craniofac Res 2020; 23: 72-81. [Crossref]

18. de Almeida-Pedrin RR, Henriques JF, de Almeida RR, de Almeida MR, McNamara JA Jr. Effects of the pendulum appliance, cervical headgear, and 2 premolar extractions followed by fixed appliances in patients with Class II malocclusion. Am J Orthod Craniofacial Orthop 2009; 136: 833-42. [Crossref]

19. Lione R, Franchi L, Laganà G, Cozza P. Effects of cervical headgear and pendulum appliance on vertical dimension in growing subjects: a retrospective controlled clinical trial. Eur J Orthod 2015; 37: 338-44. [Crossref]

20. Patel MP, Henriques JF, de Almeida RR, Pinzan A, Janson G, de Freitas MR. Comparative cephalometric study of Class II malocclusion treatment with Pendulum and Jones jig appliances followed by fixed corrective orthodontics. Dental Press J Orthod 2013; 18: 5864. [Crossref]

21. Sfondrini MF, Cacciafesta V, Sfondrini G. Upper molar distalization: A critical analysis. Orthod Craniofac Res 2002; 5: 114-26. [Crossref]

22. Kinzinger GS, Fritz UB, Sander FG, Diedrich PR. Efficiency of a pendulum appliance for molar distalization related to second and third molar eruption stage. Am J Orthod Dentofacial Orthop 2004; 125 : 8-23. [Crossref]

23. Karlsson I, Bondemark L. Intraoral maxillary molar distalization. Angle Orthod 2006; 76: 923-9. [Crossref]

24. Byloff FK, Darendeliler MA, Clar E, Darendeliler A. Distal molar movement using the pendulum appliance. Part 2: The effects of maxillary molar root uprighting bends. Angle Orthod 1997; 67: 261-70.

25. Bussick TJ, McNamara JA Jr. Dentoalveolar and skeletal changes associated with the pendulum appliance. Am J Orthod Dentofacial Orthop 2000; 117: 333-43. [Crossref]

26. Tovstein BC. Behavior of the occlusal plane and related structures in the treatment of class II malocclusion. Angle Orthod 1955; 25: 189-98.

27. Fuziy A, Rodrigues de Almeida R, Janson G, Angelieri F, Pinzan A. Sagittal, vertical, and transverse changes consequent to maxillary molar distalization with the pendulum appliance. Am J Orthod Dentofacial Orthop 2006; 130: 502-10. [Crossref]

28. Mariani L, Maino G, Caprioglio A. Skeletal versus conventional intraoral anchorage for the treatment of class II malocclusion: dentoalveolar and skeletal effects. Prog Orthod 2014; 15: 43. [Crossref]

29. Shetty S, Maurya R, Raj HVP, Patil A. Comparison of the Pendulum appliance and the Jones Jig: A prospective comparative study. Eur J Dent 2017; 11: 323-9. [Crossref]

30. Li JL, Kau C, Wang M. Changes of occlusal plane inclination after orthodontic treatment in different dentoskeletal frames. Prog Orthod 2014; 15: 41. [Crossref]

31. Tanaka EM, Sato S. Longitudinal alteration of the occlusal plane and development of different dentoskeletal frames during growth. Am J Orthod Dentofacial Orthop 2008; 134: 602.e1-602.e11. [Crossref]

32. Schudy FF. Cant of the occlusal plane and axial inclinations of teeth. Angle Orthod 1963; 33: 69-82. 